\title{
DESIGN AND TEST STUDY OF A NEW MIXED CONTROL METHOD FOR MAGNETORHEOLOGICAL SEMI-ACTIVE SUSPENSION BASED ON ELECTROMECHANICAL ANALOGY THEORY
}

\author{
Yu Jiang, Ruochen Wang, Renkai Ding, Dong Sun, Wei Liu \\ School of Automobile and Traffic Engineering, Jiangsu University, Zhenjiang 212013, China \\ e-mail:wrc@ujs.edu.cn (corresponding author: Ruochen Wang)
}

\begin{abstract}
For pursuing high performance, the development of semi-active suspension control tends to be complicated and ignores practicability. A new mixed control method effectively suppressing vibration of the vehicle body in the whole frequency band is proposed based on electromechanical analogy theory. Simulation results show that in comparison with passive suspension, on a long slope bumpy road, the mixed control reduces body acceleration by $21.49 \%$ and the maximum amplitude by $22.40 \%$. On a $\mathrm{C}$ class road, the mixed control reduces body acceleration by $9.78 \%$. Finally, an ECU hardware-in-the-loop test is conducted, which verifies the effectiveness and feasibility of the new mixed control method.
\end{abstract}

Keyword: semi-active suspension, electromechanical analogy, mixed control, hardware-in-the-loop

\section{Introduction}

Semi-active suspension can adjust the damping force or stiffness in real time according to road conditions, improving vehicle dynamic performance effectively with a low energy consumption and fast response. Therefore, such suspension has been applied in vehicles more and more widely, and has become the main research hotspot of enterprises and scholars (Tseng and Hrovat, 2015). The key problem is the design of control strategy. In 1974, Karnopp et al. (1974) first proposed a sky-hook ( $\mathrm{SH}$ ) control method for semi-active suspension. After that, the semi-active suspension control algorithm has been studied deeply, such as optimal control, neural network control, fuzzy control and so on (Bai and Lei, 2019; Liu et al., 2019; Li et al., 2019). Although the complex control algorithm can effectively improve the ride comfort of vehicles, it has high requirements on hardware and poor practicability. SH control assumes that the car body is connected to the inertial space with a damper to attenuate body vibration. It has advantages of simple logic and easy implementation, but it cannot reduce body vibration under high-frequency input (Wang et al., 2016; Ding et al., 2018). Mixed SH and ground-hook (Geng et al., 2020) assumed that the car body and tire are both connected to the inertial space with dampers, ensuring comfort and safety simultaneously, and the control effect is good in the whole frequency band. However, its control parameters need to be adjusted according to the road input, and this method is still complex. Savaresi et al. (2004) proposed Acceleration-Driven-Damper (ADD) control with a simple structure and high frequency characteristics based on the optimal theory, aiming at the problem that $\mathrm{SH}$ control is not ideal in the high frequency band. Later, he proposed a mixed SH-ADD control (Savaresi and Spelta, 2006) with good performance in the whole frequency band. However, its control flutter is large since the acceleration variable is contained in the switching equation (Liu and Zuo, 2016). A simple algorithm and good performance are the basic requirements for an engineering application. 
In this paper, the equivalent circuit model of suspension is established based on electromechanical analogy theory and a new mixed control method is proposed. Moreover, there is no acceleration variable in the mixed control switching equation, which can effectively reduce the control flutter. The equation and structural relationship between the electric circuit and mechanical vibration system are very similar (Smith, 2002; Smith and Wang, 2004). Thus, the interpretation of suspension vibration energy from the perspective of electricity can effectively broaden ideas and methods for studying the suspension system.

This study is structured as follows. Section 2 establishes the dynamic model of a $1 / 4$ semi-active suspension system and the corresponding suspension equivalent circuit based on electromechanical analogy theory. Section 3 proposes new control methods based on an electrical principle and conducts simulation analysis. Section 4 establishes the mathematical model of the magnetorheological (MR) damper and builds a real-time hardware-in-the-loop test platform. Finally, Section 5 provides a general conclusion.

\section{Semi-active suspension modeling}

The linear 1/4 semi-active suspension dynamic model is presented in Fig. 1a. Its dynamic equation can be expressed as follows

$$
\begin{aligned}
& m_{s} \dot{z}_{s}=-k_{s}\left(z_{s}-z_{u}\right)-c\left(\dot{z}_{s}-\dot{z}_{u}\right) \\
& m_{u} \dot{z}_{u}=k_{s}\left(z_{s}-z_{u}\right)-k_{u}\left(z_{u}-z_{r}\right)+c\left(\dot{z}_{s}-\dot{z}_{u}\right)
\end{aligned}
$$

where $m_{s}$ and $m_{u}$ are the sprung and unsprung masses, respectively; $z_{s}, z_{u}$ and $z_{r}$ are the vertical displacement of the sprung mass, unsprung mass and road, respectively; $k_{s}$ is the spring stiffness; $k_{u}$ is the tire stiffness; $c$ is the damping coefficient of the actual damper.

(a)

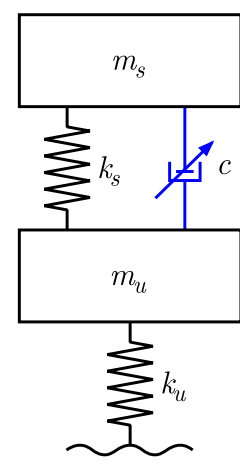

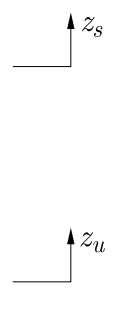

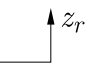

(b)

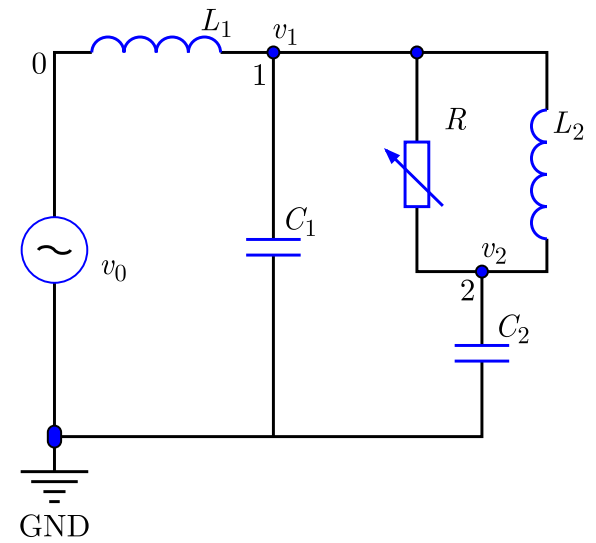

Fig. 1. (a) Suspension dynamic model, (b) Suspension equivalent circuit

According to electromechanical analogy theory (Xu et al., 2013), the $1 / 4$ semi-active suspension model can be converted to the equivalent circuit as shown in Fig. 1b. Table 1 shows the symbolic correspondence between the suspension dynamic model and the equivalent circuit. In the suspension equivalent circuit, the power supply is alternating current (AC), with changing current and voltage in real time. The electric power of components can be expressed by the product of current and voltage. The inductor and capacitor are energy storage elements. At a certain moment, their energy is not equal to electric power, but the integral of electric power over time (Makarov et al., 2016). 
Table 1. Symbol correspondence

\begin{tabular}{|l|l|}
\hline \multicolumn{1}{|c|}{ Dynamic model } & \multicolumn{1}{c|}{ Equivalent circuit } \\
\hline \hline Tire stiffness: $k_{u}$ & Inductor: $L_{1}=1 / k_{u}$ \\
\hline Spring stiffness: $k_{s}$ & Inductor: $L_{2}=1 / k_{s}$ \\
\hline Damper: $c$ & Resistor: $R=1 / c$ \\
\hline Unsprung mass: $m_{u}$ & Capacitor: $C_{1}=m_{u}$ \\
\hline Sprung mass: $m_{s}$ & Capacitor: $C_{2}=m_{s}$ \\
\hline Road excitation velocity: $\dot{z}_{r}$ & AC power: $v_{0}=\dot{z}_{r}$ \\
\hline Unsprung mass velocity: $\dot{z}_{u}$ & Voltage: $v_{1}=\dot{z}_{u}$ \\
\hline Sprung mass velocity: $\dot{z}_{s}$ & Voltage: $v_{2}=\dot{z}_{s}$ \\
\hline
\end{tabular}

For each component, the currents are

$$
\begin{aligned}
& i_{L_{1}}=\frac{1}{L_{1}} \int\left(v_{1}-v_{0}\right)=k_{u}\left(z_{u}-z_{r}\right) \quad i_{L_{2}}=\frac{1}{L_{2}} \int\left(v_{2}-v_{1}\right)=k_{s}\left(z_{s}-z_{u}\right) \\
& i_{R}=\frac{1}{R}\left(v_{2}-v_{1}\right)=c_{s}\left(\dot{z}_{s}-\dot{z}_{u}\right) \quad i_{C_{1}}=C_{1} \frac{d u}{d t}=m_{u} \ddot{z}_{u} \quad i_{C_{2}}=C_{2} \frac{d u}{d t}=m_{s} \ddot{z}_{s}
\end{aligned}
$$

Their electric power

$$
\begin{aligned}
& p_{L_{1}}=i_{L_{1}}\left(v_{1}-v_{0}\right)=k_{u}\left(z_{u}-z_{r}\right)\left(\dot{z}_{u}-\dot{z}_{r}\right) \\
& p_{L_{2}}=i_{L_{2}}\left(v_{1}-v_{0}\right)=k_{s}\left(z_{s}-z_{u}\right)\left(\dot{z}_{s}-\dot{z}_{u}\right) \\
& p_{R}=i_{R} v_{R}=R\left(v_{2}-v_{1}\right)^{2}=c\left(\dot{z}_{s}-\dot{z}_{u}\right)^{2} \\
& p_{C_{1}}=i_{C_{1}} v_{1}=m_{u} \dot{z}_{u} \ddot{z}_{u} \quad p_{C_{2}}=i_{C_{2}} v_{2}=m_{s} \dot{z}_{s} \ddot{z}_{s}
\end{aligned}
$$

Their stored electric energy

$$
\begin{aligned}
& E_{L_{1}}=\int_{0}^{t} p_{L_{1}} d t=\int_{0}^{t} i_{L_{1}} v_{L_{1}} d t=\int_{0}^{t} i_{L_{1}} L_{1} \frac{d i}{d t} d t=\frac{1}{2} L_{1} i_{L_{1}}^{2}=\frac{1}{2} k_{u}\left(z_{u}-z_{r}\right)^{2} \\
& E_{L_{2}}=\int_{0}^{t} p_{L_{2}} d t=\int_{0}^{t} i_{L_{2}} v_{L_{2}} d t=\int_{0}^{t} i_{L_{2}} L_{2} \frac{d i}{d t} d t=\frac{1}{2} L_{2} i_{L_{2}}^{2}=\frac{1}{2} k_{s}\left(z_{s}-z_{u}\right)^{2} \\
& E_{C_{1}}=\int_{0}^{t} p_{C_{1}} d t=\int_{0}^{t} i_{C_{1}} v_{1} d t=\int_{0}^{t} C_{1} v_{1} \frac{d u}{d t} d t=\frac{1}{2} C_{1} v_{1}^{2}=\frac{1}{2} m_{u} \dot{z}_{u}^{2} \\
& E_{C_{2}}=\int_{0}^{t} p_{C_{2}} d t=\int_{0}^{t} i_{C_{2}} v_{2} d t=\int_{0}^{t} C_{2} v_{2} \frac{d u}{d t} d t=\frac{1}{2} C_{2} v_{2}^{2}=\frac{1}{2} m_{s} \dot{z}_{s}^{2}
\end{aligned}
$$

\section{New mixed control method}

\subsection{Weak voltage control}

The mechanical energy of structures in the suspension system corresponds to the electric energy of the components in the equivalent circuit. The adjustable damper corresponds to sliding rheostat in the equivalent circuit. The ideal damping coefficient of the damper can be obtained by adjusting the resistance value of the sliding rheostat to reduce the electric energy of $C_{2}$. By Eq. $(2.4)_{4}$, the electric energy of $C_{2}$ depends on the absolute value of voltage $v_{2}$. Based on this, 
a weak voltage (WV) control is proposed. In the suspension equivalent circuit, the resistance of shunt complex impedance of $R$ and $L_{2}$ is

$$
|Z|=\left|\frac{Z_{R} Z_{L_{2}}}{Z_{R}+Z_{L_{2}}}\right|=\left|\frac{j w L_{2}}{j w c L_{2}+1}\right|=\frac{w L_{2}}{\sqrt{\left(w c L_{2}\right)^{2}+1}}
$$

When $v_{2}>0$ : if $v_{2}<v_{1}$, the equivalent circuit can be transformed as shown in Fig. 2a. $c$ takes the minimum value, then the shunt impedance $|Z|$ increases; the electric potential difference of the shunt resistance increases and $v_{2}$ decreases; if $v_{2}>v_{1}$, taking $C_{2}$ as the power source and $v_{2}$ as the output voltage, the transformed equivalent circuit is shown in Fig. 2b. $c$ takes the maximum value, then the shunt impedance $|Z|$ decreases; the circuit current increases; the voltage drop inside the power increases and $v_{2}$ decreases. Similarly, when $v_{2}<0$ : if $v_{2}>v_{1}$, $c$ takes the minimum value; if $v_{2}<v_{1}, c$ takes the maximum.

(a)

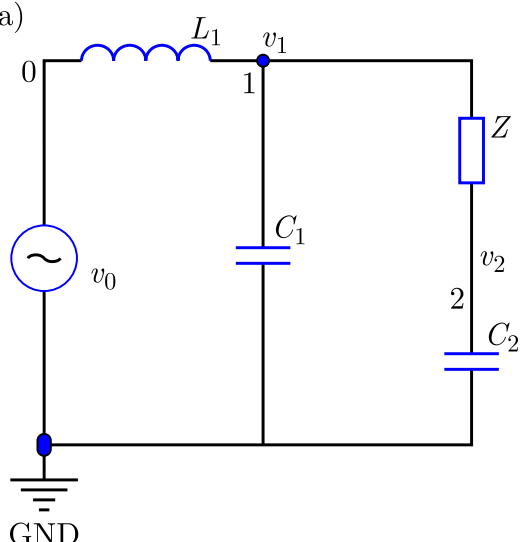

(b)

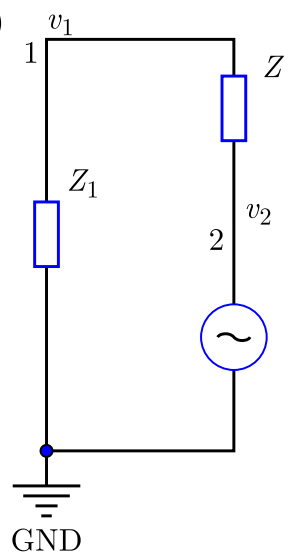

Fig. 2. Transformed equivalent circuit: (a) $v_{2}>0, v_{2}>v_{1}$, (b) $v_{2}>0, v_{2}<v_{1}$

In summary, WV control algorithm can be expressed as

$$
c_{w v}=\left\{\begin{array}{ll}
c_{\max } & v_{2}\left(v_{2}-v_{1}\right) \geqslant 0 \\
c_{\min } & v_{2}\left(v_{2}-v_{1}\right)<0
\end{array}= \begin{cases}c_{\max } & \dot{z}_{s}\left(\dot{z}_{s}-\dot{z}_{u}\right) \geqslant 0 \\
c_{\min } & \dot{z}_{s}\left(\dot{z}_{s}-\dot{z}_{u}\right)<0\end{cases}\right.
$$

\subsection{Weak current control}

Electric power represents transmitted electric energy of the components at a certain moment, corresponding to the mechanical energy instantaneously transferred by elements in the suspension system. The energy stored in the capacitor $C_{2}$ is not only small but also stable by making its electric power as zero as possible. Based on this, a weak current (WC) control is proposed. According to Kirchhoff's laws, at position 2 (Fig. 1b)

$$
i_{L_{2}}+i_{R}+i_{C_{2}}=0
$$

From Eqs. (2.2) and (3.3)

$$
\begin{aligned}
& m_{s} \ddot{z}_{s}=-k_{s}\left(z_{s}-z_{u}\right)-c\left(\dot{z}_{s}-\dot{z}_{u}\right) \\
& p_{C_{2}}=m_{s} \dot{z}_{s} \ddot{z}_{s}=-k_{s}\left(z_{s}-z_{u}\right) \dot{z}_{s}-c\left(\dot{z}_{s}-\dot{z}_{u}\right) \dot{z}_{s}
\end{aligned}
$$

Let $P_{C_{2}}=0$, then

$$
c=-\frac{k_{s}\left(z_{s}-z_{u}\right)}{\left(\dot{z}_{s}-\dot{z}_{u}\right)}
$$


When $z_{s}-z_{u}$ is different from $\dot{z}_{s}-\dot{z}_{u}$, the above equation is true. If $z_{s}-z_{u}$ and $\dot{z}_{s}-\dot{z}_{u}$ have the same sign, in order for $P_{C_{2}}$ to approach zero, $c$ must be minimized. In summary, WC control algorithm can be expressed as

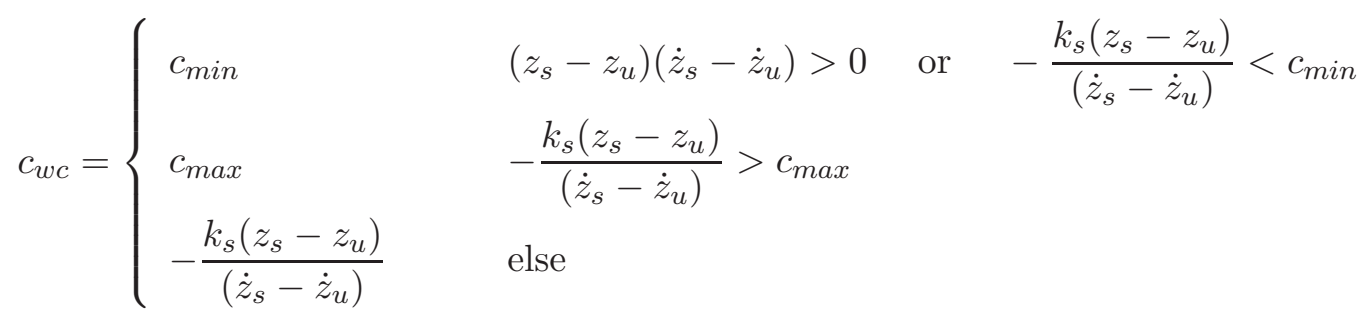

\subsection{Mixed control}

A mixed WV and WC control is proposed in this Section. In order to clear the transferred energy of $C_{2}$ and other components, the AC power $v_{0}, L_{1}$ and $C_{1}$ in the suspension equivalent circuit are regarded as the power $v_{1}$ with the equivalent resistor $R_{1}$ as shown in Fig. 3.

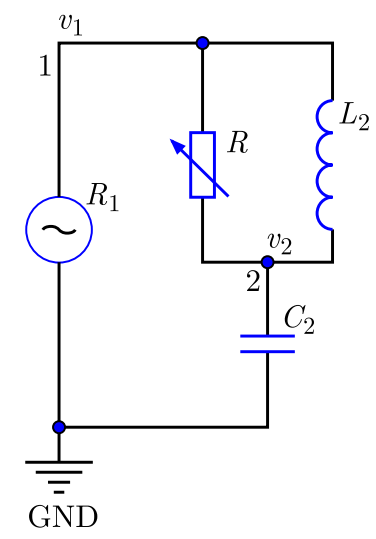

Fig. 3. Transformed equivalent circuit with power $v_{1}$

The electric power of $R_{1}$ and $C_{2}$ is

$$
\begin{aligned}
& p_{R_{1}}=I v_{1}=-k_{s}\left(z_{s}-z_{u}\right) \dot{z}_{u}-c\left(\dot{z}_{s}-\dot{z}_{u}\right) \dot{z}_{u} \\
& p_{C_{2}}=I v_{2}=-k_{s}\left(z_{s}-z_{u}\right) \dot{z}_{s}-c\left(\dot{z}_{s}-\dot{z}_{u}\right) \dot{z}_{s}
\end{aligned}
$$

where $k_{s}\left(z_{s}-z_{u}\right) \dot{z}_{s}$ and $k_{s}\left(z_{s}-z_{u}\right) \dot{z}_{u}$ represent the electric power transmitted by $L_{2}$ to $C_{2}$ and $R_{1}$. $c\left(\dot{z}_{s}-\dot{z}_{u}\right) \dot{z}_{s}$ and $c\left(\dot{z}_{s}-\dot{z}_{u}\right) \dot{z}_{u}$ represent the electric power transmitted by $R$ to $C_{2}$ and $R_{1} . L_{2}$ is not analyzed as it is an energy storage element with a fixed inductance value. The sum of the electric power transferred by $R$ to the $C_{2}$ and $R_{1}$ is

$$
p_{C_{2} R_{1}}=c\left(\dot{z}_{s}-\dot{z}_{u}\right) \dot{z}_{s}+c\left(\dot{z}_{s}-\dot{z}_{u}\right) \dot{z}_{u}=c\left(\dot{z}_{s}^{2}-\dot{z}_{u}^{2}\right)
$$

If $P_{C_{2} R_{1}} \geqslant 0$, it indicates that the electric power transmitted by $R$ to $C_{2}$ is greater than $R_{1}$, and the power can be better dissipated out from $C_{2}$. WV control is selected. If $P_{C_{2} R_{1}}<0$, the electric power transmitted by $R$ to $C_{2}$ is less than $R_{1}$, and the dissipation capacity of $C_{2}$ is poor. At this time, WC control is chosen to make $C_{2}$ short circuit, hence $R$ and $L_{2}$ can absorb as much electric power as possible. In summary, the mixed control algorithm can be expressed as

$$
c_{\text {mix }}= \begin{cases}c_{w v} & \dot{z}_{s}^{2}-\dot{z}_{u}^{2} \geqslant 0 \\ c_{w c} & \dot{z}_{s}^{2}-\dot{z}_{u}^{2}<0\end{cases}
$$




\subsection{Simulation analysis}

MR damper is selected as the semi-active suspension actuator, and its mathematical model is built in Section 4.1. Taking the passive damper and MR damper with the SH-ADD control method as a comparison, the dynamic performance of the mixed control is analyzed. The specific suspension system parameters are shown in Table 2.

Table 2. System parameters

\begin{tabular}{|c|c|c|c|}
\hline Symbols & Description & Values & Units \\
\hline \hline$m_{s}$ & Sprung mass & 317.5 & $\mathrm{Kg}$ \\
\hline$m_{u}$ & Unsprung mass & 45.4 & $\mathrm{Kg}$ \\
\hline$k_{s}$ & Spring stiffness & 20000 & $\mathrm{~N} / \mathrm{m}$ \\
\hline$k_{u}$ & Wheel stiffness & 192000 & $\mathrm{~N} / \mathrm{m}$ \\
\hline$c_{p}$ & Passive damping & 1600 & $\mathrm{Ns} / \mathrm{m}$ \\
\hline
\end{tabular}

(a)

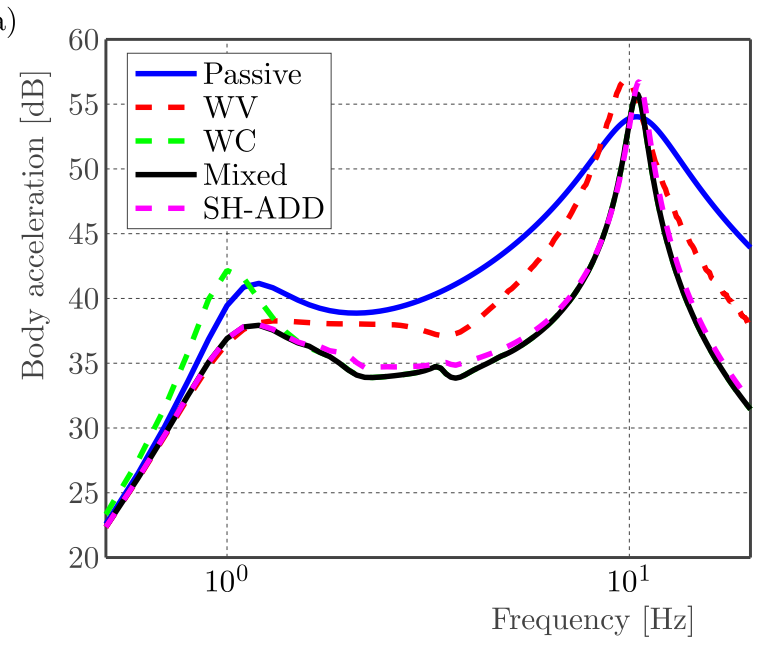

(b)

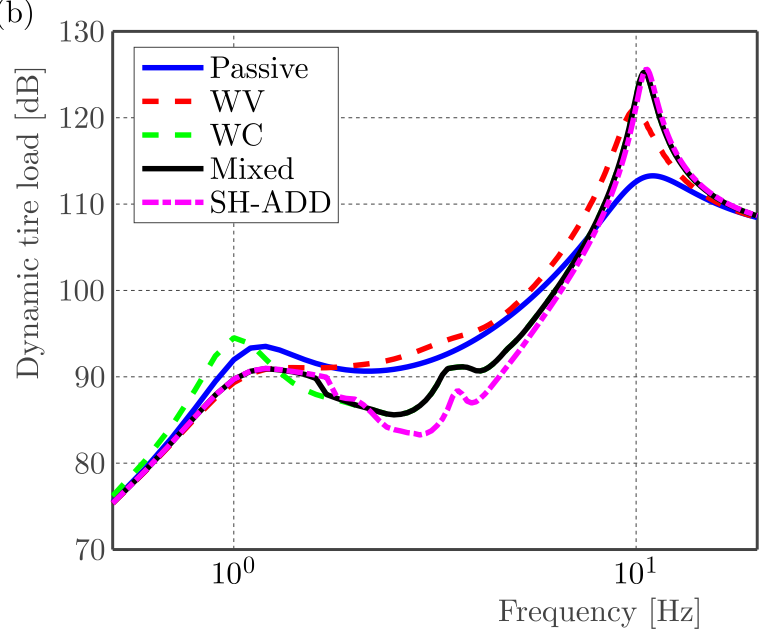

(c)

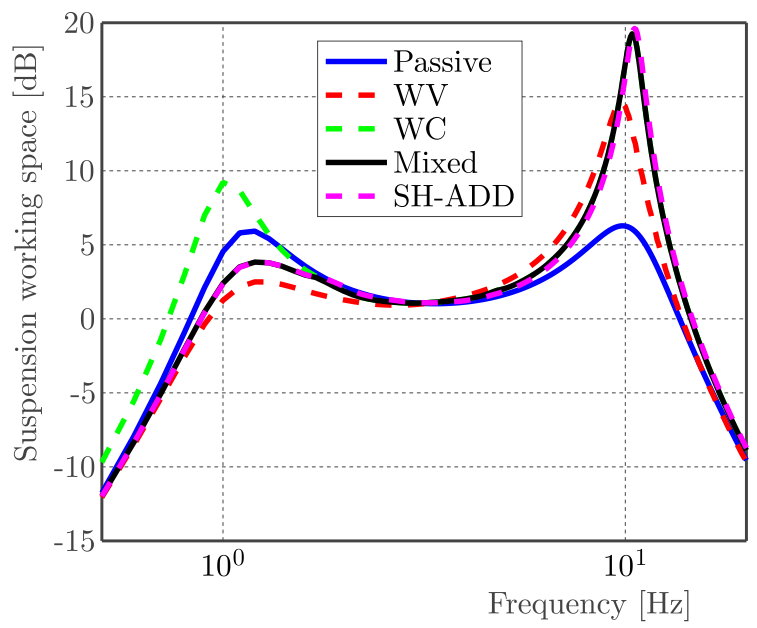

Fig. 4. Amplitude-frequency characteristics

Figure 4 shows the amplitude-frequency characteristics of dynamic responses. From Fig. 4a, $\mathrm{WV}$ can effectively reduce body acceleration in the low frequency band and WC can effectively reduce in the high one. The mixed control integrates the filtering characteristics of WV and $\mathrm{WC}$, having excellent performance in the whole frequency band. Compared with the SH-ADD 
control, possessing similar performance, the mixed control has a better effect in the middle and high frequency band. From Fig. 4b, the mixed control benefits the tire dynamic load both in the middle and low frequency band, but deteriorates in the high band, while the SH-ADD control has better performance in the middle frequency band than the mixed control. As seen in Fig. 4c, the mixed control and SH-ADD control have roughly the same influence on the suspension working space, being good in the low frequency band but worsening in the high range.

To study the improvement of the vehicle dynamic performance under the new mixed control method, this paper establishes two typical roads, namely, long slope bumpy road and the C class stochastic road. The long slope bumpy road mainly simulates the speed of the bump. According to GB/T5902-1986 vehicle ride test method of impulse input for ride comfort, the long slope bumpy road should meet the following formula

$$
q=\frac{1}{100}\left(1-\cos \frac{2 \pi x}{5000}\right) \quad 0<x<5000
$$

where $x$ is the distance in the forward direction; $q$ is the road height; unit in millimeters.

The stochastic road model (Meng et al., 2020) is expressed as follows

$$
\dot{z}_{r}=-2 \pi f_{0} z_{r}+2 \pi n_{0} w \sqrt{G_{q}\left(n_{0}\right) v}
$$

where $f_{0}=n_{00} v$ is the cut-off frequency $\left(n_{00}=0.011 \mathrm{~m}^{-1}\right.$ is the space cut-off frequency), $n_{0}$ is the space frequency which equals $0.1 \mathrm{~m}^{-1}, v$ is the vehicle velocity, $w$ is the Gaussian white noise with a zero-mean-value, and $G_{q}\left(n_{0}\right)$ is the road roughness coefficient. According to the international standard ISO 8608 , the roughness coefficients of the $\mathrm{C}$ class road is $256 \cdot 10^{-6} \mathrm{~m}^{-3}$.

The inputs of the long slope bumpy road and the C class road at a speed of $10 \mathrm{~m} / \mathrm{s}$ are shown in Fig. 5. Figure 6 shows the time-domain dynamic responses including body acceleration, tire dynamic load and suspension working space under the mixed and SH-ADD control.

(a)

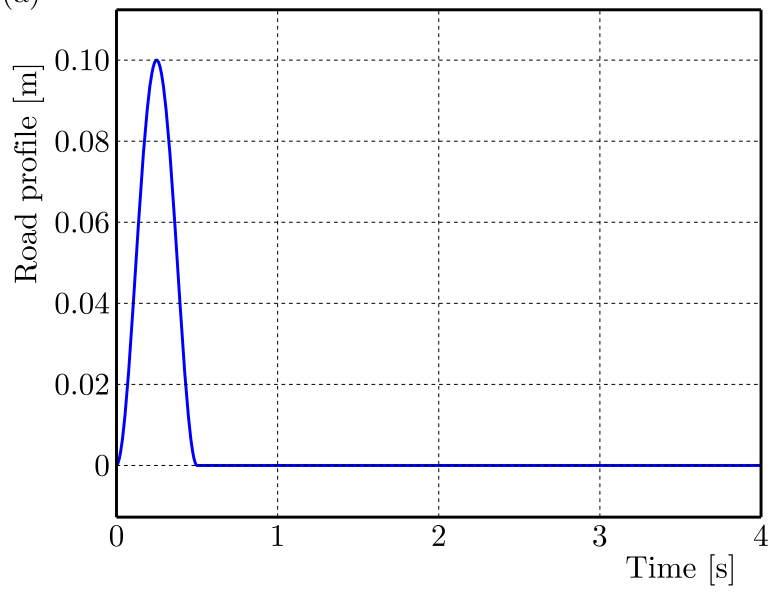

(b)

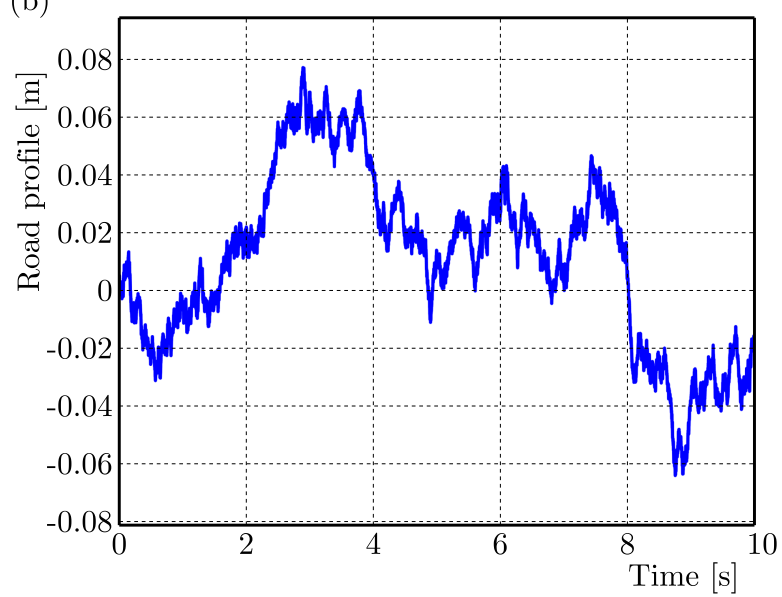

Fig. 5. Road input: (a) long slope bumpy road, (b) C class road

Compared with passive suspension, on a long slope bumpy road, the mixed control obviously accelerates the response speed of body acceleration. And the mixed control decreases body acceleration by $21.49 \%$ and the maximum amplitude by $22.40 \%$, while SH-ADD reduces by $18.18 \%$ and $16.67 \%$, respectively. The mixed control and SH-ADD have certain optimization of the tire dynamic load. On the $\mathrm{C}$ class road, the mixed control reduces body acceleration by $9.78 \%$ and SH-ADD reduces by $7.61 \%$, compared with the passive suspension. Although the mixed control deteriorates the tire dynamic load and suspension working space, it is within an acceptable range. Generally, the mixed control can improve ride comfort effectively. The specific root mean square (RMS) values of each response index are shown in Table 3. 
(a)
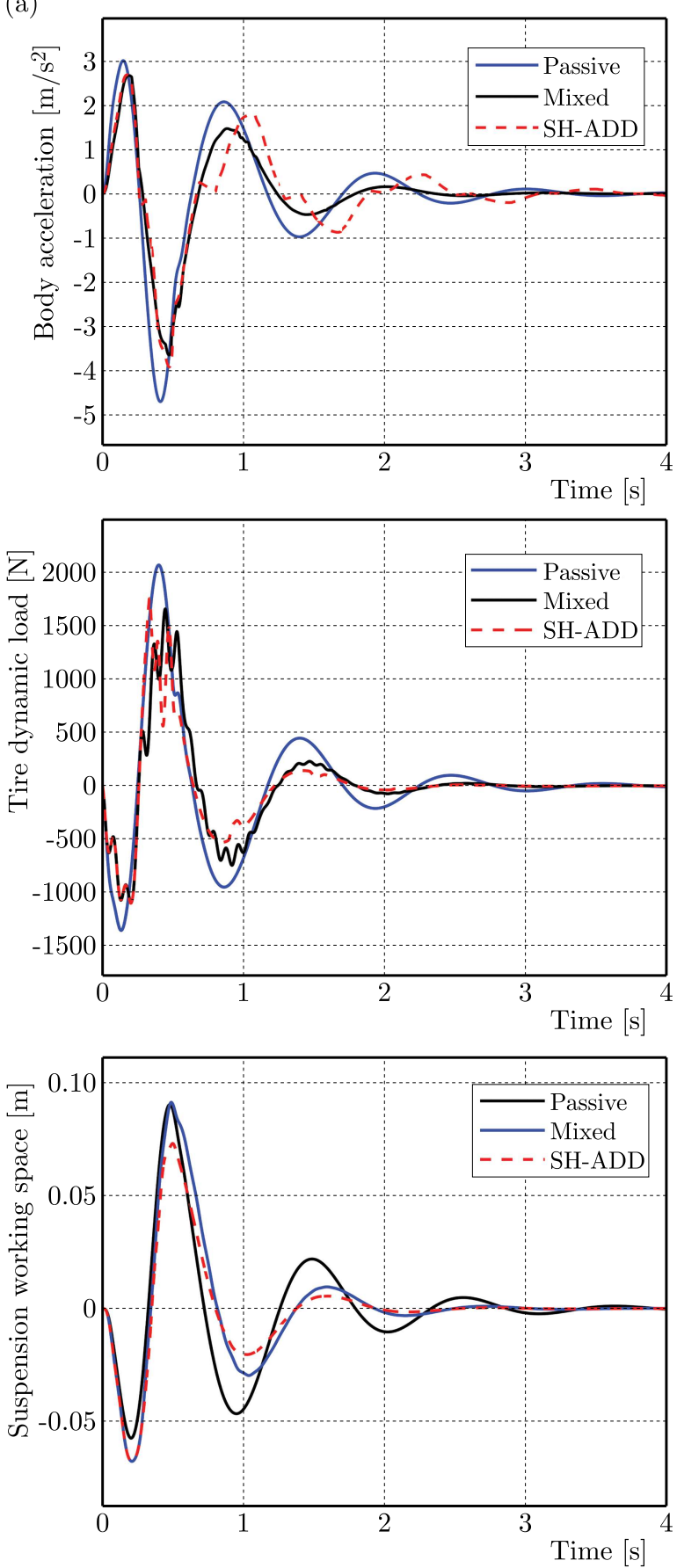

(b)
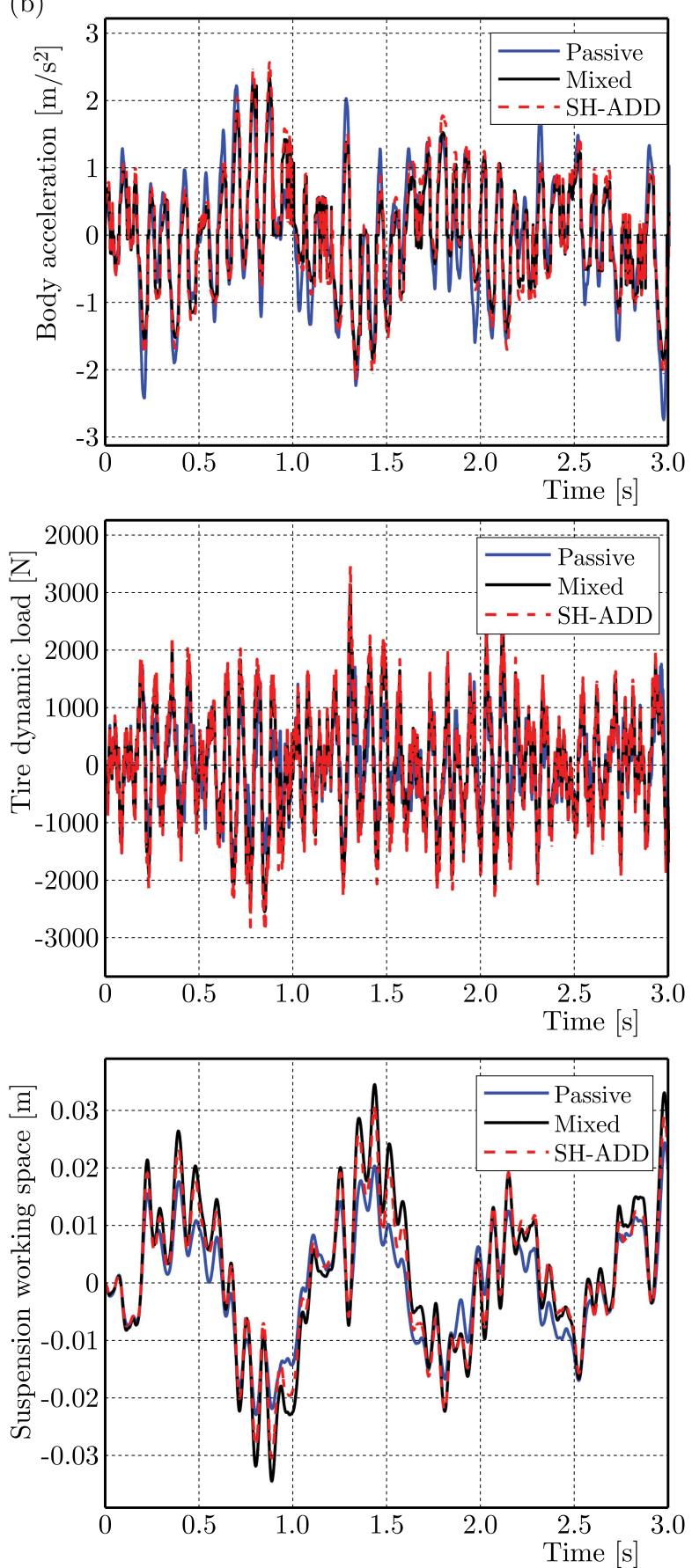

Fig. 6. Time-domain responses: (a) on long slope bumpy road, (b) on $\mathrm{C}$ class road

Table 3. RMS of three indexes

\begin{tabular}{|c|c|c|c|c|c|c|}
\hline & \multicolumn{2}{|c|}{ Long slope bump road } & \multicolumn{3}{c|}{ C class road } \\
\cline { 2 - 7 } & $\begin{array}{c}\text { Body } \\
\text { acceleration } \\
{\left[\mathrm{m} / \mathrm{s}^{2}\right]}\end{array}$ & $\begin{array}{c}\text { Tire } \\
\text { dynamic } \\
\text { load }[\mathrm{N}]\end{array}$ & $\begin{array}{c}\text { Suspension } \\
\text { working } \\
\text { space }[\mathrm{m}]\end{array}$ & $\begin{array}{c}\text { Body } \\
\text { acceleration } \\
{\left[\mathrm{m} / \mathrm{s}^{2}\right]}\end{array}$ & $\begin{array}{c}\text { Tire } \\
\text { dynamic } \\
\text { load }[\mathrm{N}]\end{array}$ & $\begin{array}{c}\text { Suspension } \\
\text { working } \\
\text { space }[\mathrm{m}]\end{array}$ \\
\hline \hline Passive & 1.21 & 546.1 & 0.0254 & 0.92 & 710.6 & 0.0116 \\
\hline SH-ADD & 0.99 & 385.8 & 0.0215 & 0.85 & 851.2 & 0.0138 \\
\hline Mixed & 0.95 & 415.4 & 0.0256 & 0.83 & 844.9 & 0.0139 \\
\hline
\end{tabular}




\section{Test verification}

\subsection{Inverse model of MR damper}

Establishing an accurate inverse model is the precondition of precise control for the MR damper. Therefore, the polynomial model (Ding et al., 2017, 2019) is applied to construct the inverse model of the MR damper in this paper. Choi et al. (2001) divided the hysteresis loop of velocity-force characteristics into two regions: positive acceleration (lower loop) and negative acceleration (upper loop). A fractional polynomial of the 5th order numerator and 2nd order denominator is used to fit the velocity characteristics, and the 2 nd order fitting of $a_{i}$ is carried out to improve the fitting accuracy. Hence, the damping force of the MR damper can be expressed by the following

$$
F=\frac{1}{v^{2}+a_{6} v+a_{7}} \sum_{i=0}^{5} a_{i} v^{i}
$$

where $a_{i}=b_{i} I^{2}+c_{i} I+d_{i}(i=0,1, \ldots, 7) ; v$ is the suspension velocity.

Form Eq. (4.1), the control current can be obtained

$$
\begin{aligned}
& \left(F_{\text {ideal }} b_{6} v^{2}+F_{\text {ideal }} b_{7}-\sum_{i=0}^{5} b_{i} v^{i}\right) I^{2}+\left(F_{\text {ideal }} c_{6}+F_{\text {ideal }} c^{7}-\sum_{i=0}^{5} c_{i} v^{i}\right) I \\
& \quad+F_{\text {ideal }} v^{2}+d_{6} v+d_{7}-\sum_{i=0}^{5} d_{i} v^{i}=0
\end{aligned}
$$

where $F_{\text {ideal }}$ is the ideal damping force.

The key to improving the accuracy of the polynomial model is the polynomial coefficient. Therefore, the external characteristic test of the MR damper must be conducted. The characteristics of the MR damper are obtained by loading the control current from $0 \mathrm{~A}$ to $3.5 \mathrm{~A}$ at an interval of $0.25 \mathrm{~A}$, as shown in Fig. 7 .

(a)

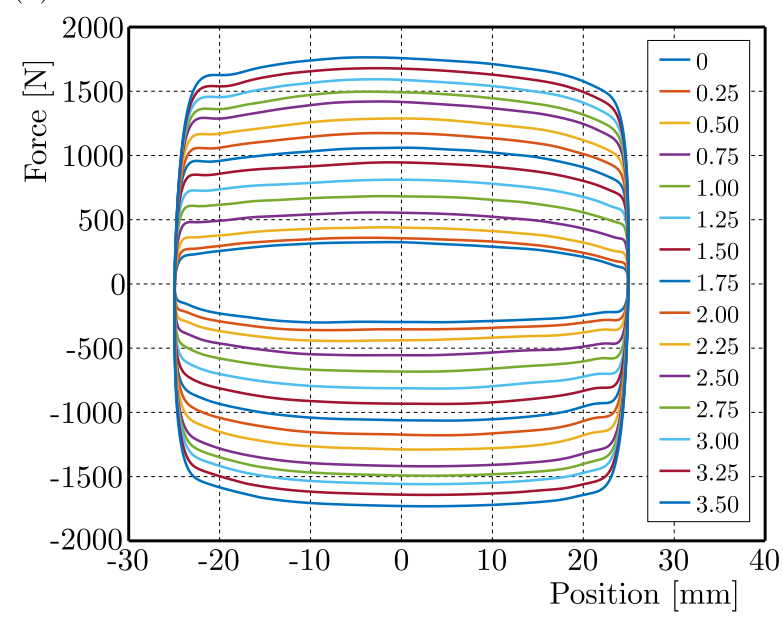

(b)

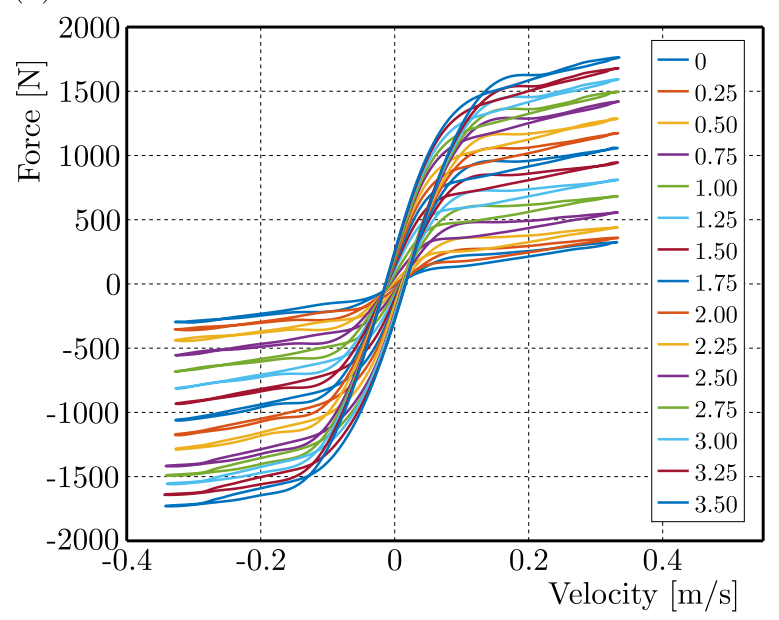

Fig. 7. Characteristics of MR damper: (a) position-force, (b) velocity-force

The fitting effects are shown in Fig. 8.

\subsection{Hardware-in-the-loop test}

An ECU hardware-in-the-loop test system is built based on xPC Target to verify the feasibility of the proposed control method, as shown in Fig. 9. xPC Target adopts the dual-machine 
(a)

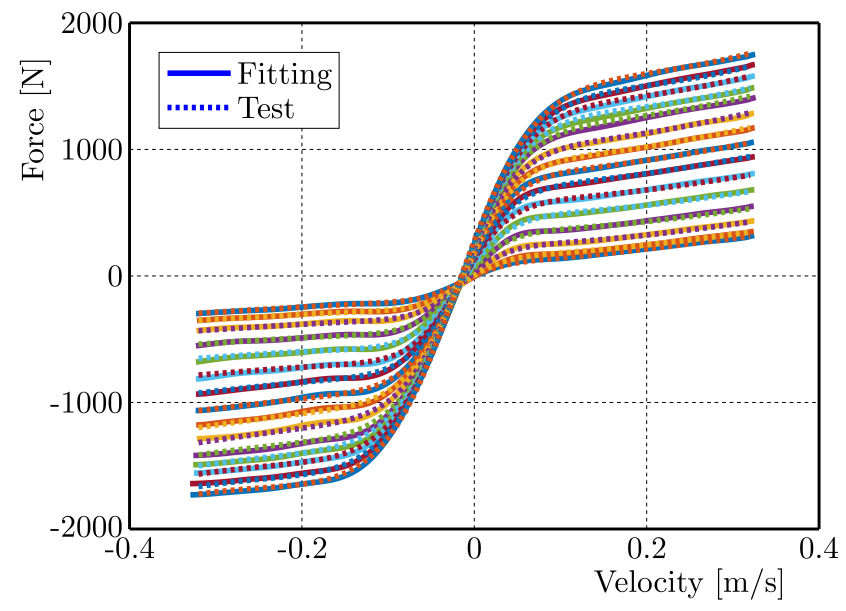

(b)

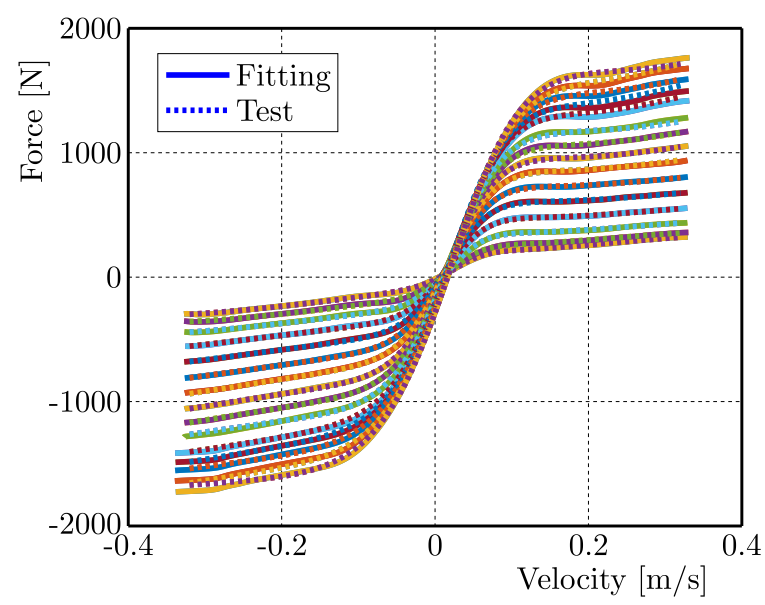

Fig. 8. Fitting effects: (a) upper loop, (b) lower loop

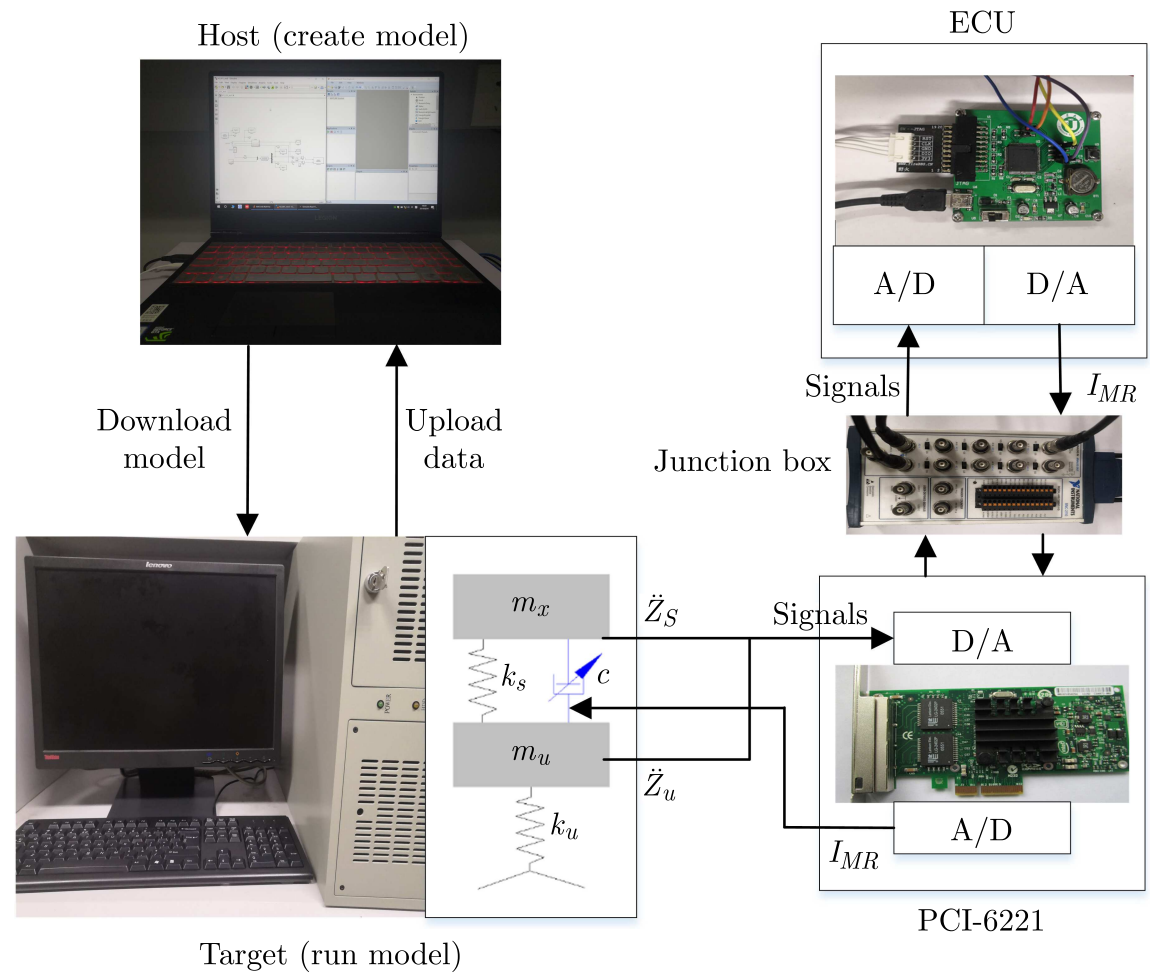

Fig. 9. Test configuration

mode of a target-host computer. The host establishes a suspension Simulink model, defines the input and output signals, and generates the model code. The target runs the xPC Target real-time kernel, runs the suspension model and outputs the state signals (Hristu-Varsakelis and Levine, 2005; Bi and Yang, 2014). The host computer communicates with the target through the Ethernet TCP/IP protocol, and the C code generated by the host is downloaded to the target via Ethernet. ECU reveives suspension acceleration signals through a digital acquisition board (NI PCI-6221), runs the control algorithm and the inverse model program of the MR damper, and outputs the control current to the target machine. In addition, they are connected by the BNC-2110 junction box with a shielded outer box effectively reducing the noise interference. Thus, the ECU hardware-in-the-loop closed loop control test is completed. 
Test results show that on a long slope bumpy road, compared with passive suspension, the mixed control reduces body acceleration by $18.19 \%$ and the maximum amplitude by $19.63 \%$, while the SH-ADD reduces by $13.50 \%$ and $12.01 \%$, respectively. On the C class road, the mixed control reduces body acceleration by $5.93 \%$ and SH-ADD reduces by $4.26 \%$. Figure 10 shows a comparison of the simulation and test for vehicle body acceleration on two roads. The errors are $5.05 \%$ and $4.26 \%$, respectively, which are within an acceptable range. The comparison of RMS values of dynamic responses is shown in Figs. 11 and 12. Although there are some errors between the test and simulation results, in general, the performance of the control algorithm in the real controller keeps high consistency with the simulation, which verifies the correctness and effectiveness of the proposed control method.

(a)

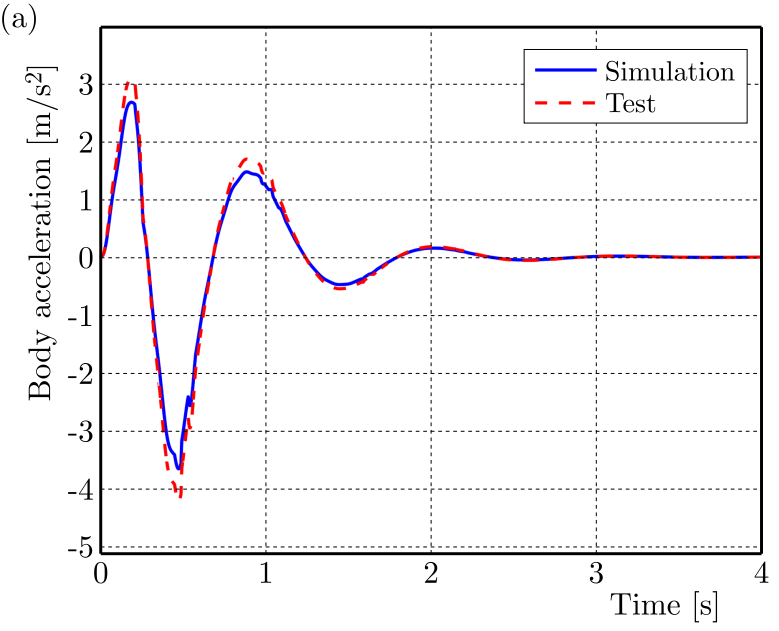

(b)

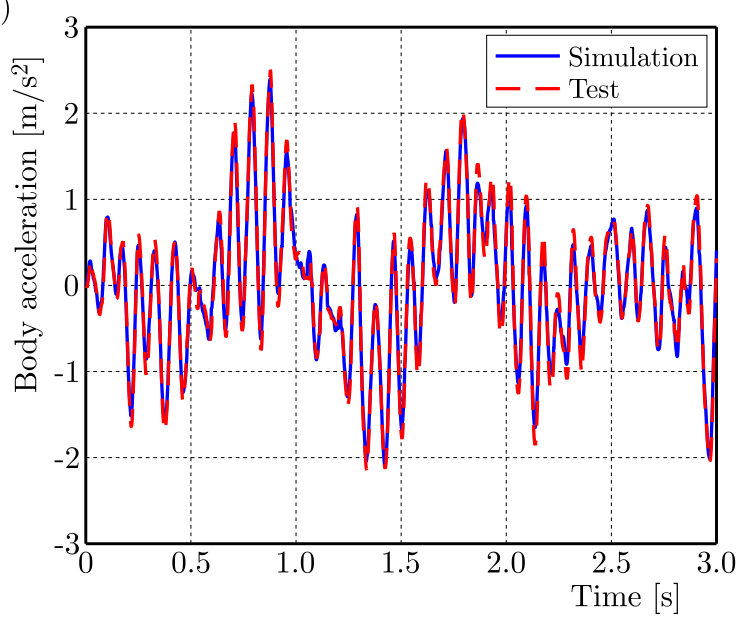

Fig. 10. Comparison of body acceleration: (a) on the long slope bumpy road, (b) on the C class road
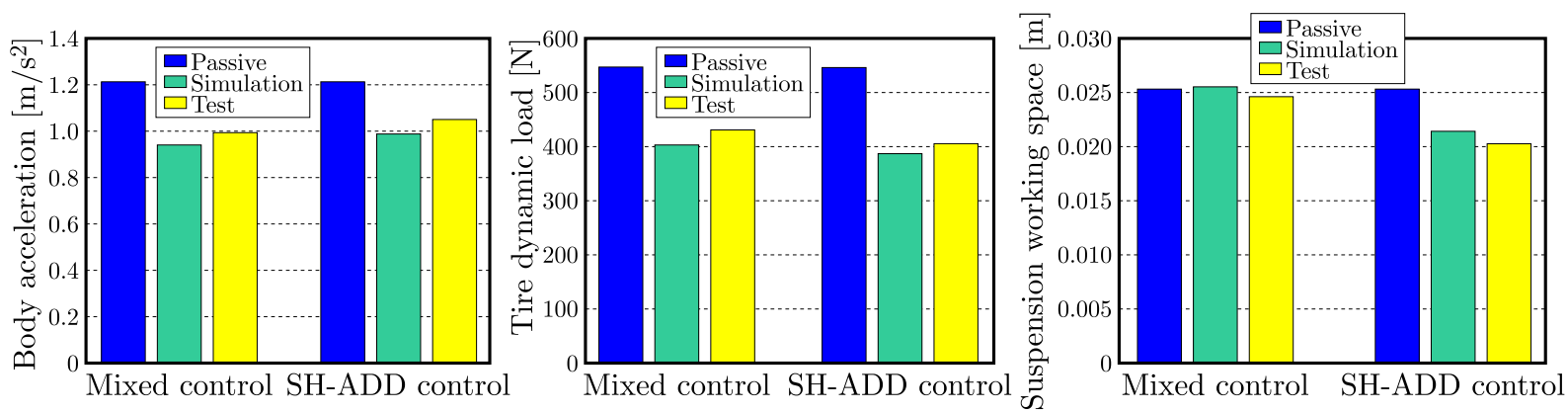

Fig. 11. Comparison of simulation and test on the long slope bumpy road
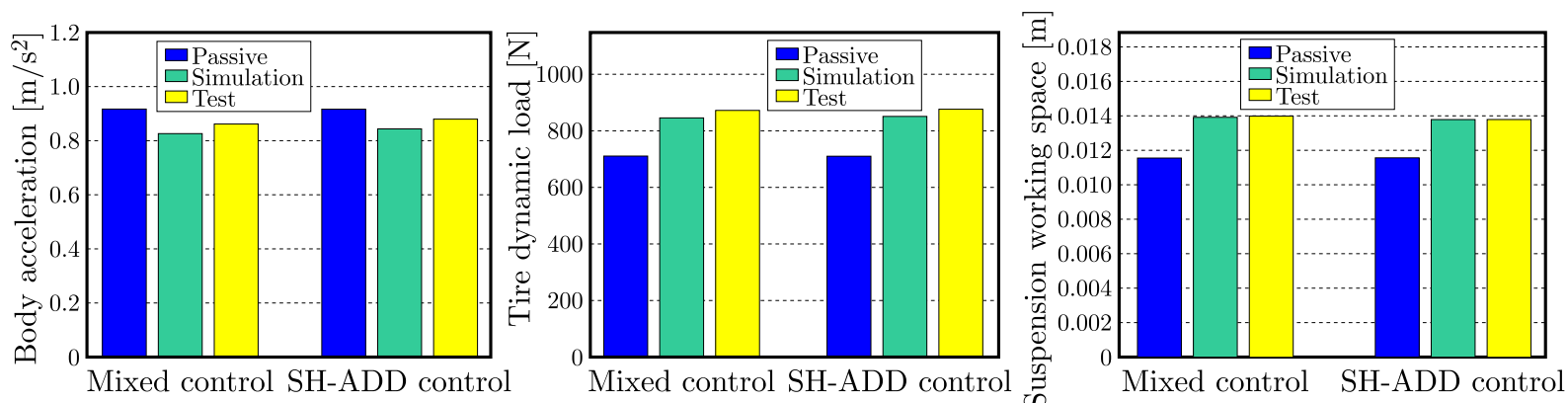

Fig. 12. Comparison of simulation and test on the $\mathrm{C}$ class road 


\section{Conclusion}

In this paper, a new semi-active mixed control method is proposed from the electrical perspective. The correctness and validity of the control method are verified by an ECU hardware-in-the-loop test. The main conclusions are summarized as follows:

- WV control can effectively suppress body vibration in the low frequency band, and WC control performs good in the high. The new mixed control combines the great characteristics of the two, having excellent performance in the whole frequency band.

- The simulation results show that in comparison with passive suspension, on the long slope bumpy road, the mixed control reduces body acceleration by $21.49 \%$ and the maximum amplitude by $22.40 \%$, while SH-ADD decreases by $18.18 \%$ and $16.67 \%$, respectively. On the $\mathrm{C}$ class road, the mixed control reduces body acceleration by $9.78 \%$ and SH-ADD decreases by $7.61 \%$. The ride comfort performance of the mixed control is better than that of SH-ADD.

- An ECU hardware-in-the-loop test is conducted based on the xPC Target. In general, the performance in a real controller keeps high consistency with simulation, which verifies the effectiveness and feasibility of the proposed control method.

\section{Acknowledgement}

This work was supported by National Natural Science Foundation of China (grant No. 51975253).

\section{References}

1. BAI X.-L., LeI J., 2019, Internal model-based optimal vibration control for linear vehicle suspension systems with actuator delay, Ferroelectrics, 549, 1, 195-203

2. Bi Y.L., YANG D., 2014, Development of interface card drivers based on matlab/xPC Target, Key Engineering Materials, 620, 563-568

3. Choi S.B., LeE S.K., PARK Y.P., 2001, A hysteresis model for the field-dependent damping force of a magnetorheological damper, Journal of Sound and Vibration, 245, 2, 375-383

4. Ding R., Wang R., Meng X., Chen L., 2017, Study on coordinated control of the energy regeneration and the vibration isolation in a hybrid electromagnetic suspension, Proceedings of the Institution of Mechanical Engineers, Part D: Journal of Automobile Engineering, 231, 11, 1530-1539

5. Ding R., Wang R., Meng X., Chen L., 2018, A modified energy-saving skyhook for active suspension based on a hybrid electromagnetic actuator, Journal of Vibration and Control, 25, 2, $286-297$

6. Ding R., Wang R., Meng X., Chen L., 2019, Energy consumption sensitivity analysis and energy-reduction control of hybrid electromagnetic active suspension, Mechanical Systems and Signal Processing, 134, 106301

7. Geng G., Yu Y., Sun L., Li H., 2020, Research on ride comfort and driving safety under hybrid damping extension control for suspension systems, Applied Sciences-Basel, 10, 4, 1442

8. Hristu-Varsakelis D., Levine W.S., 2005, Handbook of Networked and Embedded Control Systems, Birkhäuser Boston

9. Karnopp D., Crosby M.J., Harwood R.A., 1974, Vibration control using semi-active suspension control, Journal of Engineering for Industry, 96, 619-626

10. Li H., Zhang Z., YAn H., Xie X., 2019, Adaptive event-triggered fuzzy control for uncertain active suspension systems, IEEE Transactions on Cybernetics, 49, 12, 4388-4397 
11. Liu Y., Zeng Q., Tong S., Chen C.L.P., Liu L., 2019, Adaptive neural network control for active suspension systems with time-varying vertical displacement and speed constraints, IEEE Transactions on Industrial Electronics, 66, 12, 9458-9466

12. Liu Y., Zuo L., 2016, Mixed skyhook and Power-Driven-Damper: a new low-jerk semi-active suspension control based on power flow analysis, Journal of Dynamic Systems, Measurement, and Control, 138, 8

13. Meng X., Ding R., Sun Z., Wang R., Chen L., 2020, Multi-mode switching control of hybrid electromagnetic suspension based on road conditions adaptation, Journal of Theoretical and Applied Mechanics, 58, 3, 697-710

14. Makarov S.N., Ludwig R., Bitar S.J., 2016, Practical Electrical Engineering, DOI: 10.1007/ 978-3-319-21173-2

15. Savaresi S.M., Silani E., Bittanti S., 2004, Acceleration-Driven-Damper (ADD): an optimal control algorithm for comfort-oriented semiactive suspensions, Journal of Dynamic Systems, Measurement, and Control, 127, 2, 218-229

16. Savaresi S.M., Spelta C., 2006, Mixed sky-hook and ADD: approaching the filtering limits of a semi-active suspension, Journal of Dynamic Systems, Measurement, and Control, 129, 4, 382-392

17. SмIтH M.C., 2002, Synthesis of mechanical networks: the inerter, IEEE Transactions on Automatic Control, 47, 10, 1648-1662

18. Sмith M.C., Wang F.-C., 2004, Performance benefits in passive vehicle suspensions employing inerters, Vehicle System Dynamics, 42, 4, 235-257

19. Tseng H.E., Hrovat D., 2015, State of the art survey: active and semi-active suspension control, Vehicle System Dynamics, 53, 7, 1034-1062

20. Wang R., Ding R., Chen L., 2016, Application of hybrid electromagnetic suspension in vibration energy regeneration and active control, Journal of Vibration and Control, 24, 1, 223-233

21. Xu X., Jiang H., GaO M.H., 2013, Modeling and validation of air suspension with auxiliary chamber based on electromechanical analogy theory, Applied Mechanics and Materials, 437, 190-193 